\title{
Correlation structure regularization via entropy loss function for high-dimension and low-sample-size data
}

DOI:

10.1080/03610918.2019.1571607

\section{Document Version}

Accepted author manuscript

Link to publication record in Manchester Research Explorer

\section{Citation for published version (APA):}

Chen, C., Zhou, J., \& Pan, J. (2019). Correlation structure regularization via entropy loss function for highdimension and low-sample-size data. COMMUNICATIONS IN STATISTICS-SIMULATION AND COMPUTATION, 1-16. https://doi.org/10.1080/03610918.2019.1571607

\section{Published in:}

COMMUNICATIONS IN STATISTICS-SIMULATION AND COMPUTATION

\section{Citing this paper}

Please note that where the full-text provided on Manchester Research Explorer is the Author Accepted Manuscript or Proof version this may differ from the final Published version. If citing, it is advised that you check and use the publisher's definitive version.

\section{General rights}

Copyright and moral rights for the publications made accessible in the Research Explorer are retained by the authors and/or other copyright owners and it is a condition of accessing publications that users recognise and abide by the legal requirements associated with these rights.

\section{Takedown policy}

If you believe that this document breaches copyright please refer to the University of Manchester's Takedown Procedures [http://man.ac.uk/04Y6Bo] or contact uml.scholarlycommunications@manchester.ac.uk providing relevant details, so we can investigate your claim.

\section{OPEN ACCESS}




\title{
Correlation structure regularization via entropy loss function for high-dimension and low-sample-size data
}

\author{
Chen Chen ${ }^{\mathrm{a}}$, Jie Zhou ${ }^{\mathrm{a} *}$, Jianxin Pan $^{\mathrm{b}}$ \\ ${ }^{a}$ College of Mathematics, Sichuan University, Chengdu, Sichuan 610064, China \\ ${ }^{b}$ School of Mathematics, University of Manchester, Manchester M13 9PL, UK
}

\begin{abstract}
Estimating structured covariance or correlation matrix has been paid more and more attentions in recent years. A recent method based on the entropy loss function was proposed to regularize the covariance structure for a given covariance matrix whose underlying structure may be blurred due to random noises from different sources. However, the entropy loss function considered is very likely to be unavailable in covariance regularization for high-dimension and low-sample-size (HDLSS) data. In this paper, a new discrepancy is proposed for regularizing correlation structure, in which the given correlation matrix (e.g., sample correlation matrix) and the candidate structure in the entropy loss function are both added by the identity matrix multiplied by a constant, so that the problem owing to likely singularity of sample correlation matrix for HDLSS data can be overcome. The candidate correlation structures considered in this paper include tri-diagonal Toeplitz, compound symmetry, AR(1) and banded Toeplitz. The regularized correlation estimates for the first three structures can be obtained by solving one-dimensional optimization problems, while the regularized one for the fourth structure can be computed efficiently using Newton's iteration method. Simulation studies show that the proposed new approach works well, providing a reliable method to regularize the correlation structure for HDLSS data.
\end{abstract}

Keywords: Bregman divergence; Correlation matrix estimation; Entropy loss function; High-dimensional correlation matrix; Regularization

\section{Introduction}

The covariance matrices with certain special structures have emerged widely in various application fields such as signal processing (Pascal et al., 2008; Soloveychik and Wiesel, 2014), ultrasound imaging (Asl and Mahloojifar, 2012), neuroimaging (Zhou et al., 2016), genetics selection (Meyer, 2009), and social science (van der Leeden et al., 1996). It is usually necessary to estimate the structured covariance or correlation matrix whose underlying structure may be blurred by random noise. This problem was studied by many authors in the literature (see, e.g., Kang et al. (2015); Soloveychik et al. (2016); Sun et al. (2015) and references therein). Estimating structured covari-

*Corresponding author. E-mail: jzhou@scu.edu.cn 
ance matrix with high-dimension is fundamental in statistics. Lots of existing works in statistics provided various estimators for structured covariance matrices, including the maximum likelihoodbased estimators (Wang and Carey, 2003; Jennrich and Schluchter, 1986; Daniels and Kass, 2001; Feng et al., 2016) and the minimum distance estimators in the sense of minimizing the Frobenius or spectral norm of the estimation error matrix (Kim and Lee, 2013; Shults and Chaganty, 1998). A novel method proposed in Lin et al. (2014) that regularizes the underlying covariance structure of a given covariance matrix is based on the entropy loss function

$$
L(A, R)=\operatorname{tr}\left(A^{-1} R\right)-\log \left(\operatorname{det}\left(A^{-1} R\right)\right)-p,
$$

the Kullback-Leibler (KL) divergence from $p \times p$ matrix $A$ to $p \times p$ matrix $R$. Note that the KL divergence is not a strict distance measure in mathematics because it is not symmetric and does not obey the triangle inequality. In Lin et al. (2014), a class of candidate covariance structures including tri-diagonal Toeplitz, compound symmetry, AR(1) and banded Toeplitz were considered. Denote $\mathcal{P}$ as the set of all positive definite correlation matrices with a certain structure in some available class. The regularization is then made by calculating the discrepancy between a given symmetric positive definite covariance matrix $A$ and the set $\mathcal{P}$ :

$$
D(A, \mathcal{P})=\min _{R \in \mathcal{P}} L(A, R)
$$

When taking the matrix $A$ as the sample covariance or correlation matrix, however, this method becomes numerically unstable in the case that the sample size $n$ and the dimension $p$ are close even if $n$ is larger than $p$. It quickly becomes unavailable when $p$ is larger than $n$ owing to the singularity of the matrix $A$.

To overcome the shortage of the proposed method in Lin et al. (2014), an improved regularization method is proposed in this paper, which is based on the entropy loss function between $p \times p$ matrices $A+\lambda I$ and $R+\lambda I$ :

$$
\Delta_{\lambda}(A, R)=\operatorname{tr}\left((A+\lambda I)^{-1}(R+\lambda I)\right)-\log \operatorname{det}\left((A+\lambda I)^{-1}(R+\lambda I)\right)-p, \quad \lambda>0,
$$

where $I$ is $p \times p$ identity matrix. Note that here we consider $\Delta_{\lambda}(A, R)$ as a newly proposed loss function between matrices $A$ and $R$, not between matrices $A+\lambda I$ and $R+\lambda I$. In this paper, we are interested in correlation structure regularization, then the matrices $A$ and $R$ are respectively the given correlation matrix and unknown but structured correlation matrix. In the following, instead of using the discrepancy defined by (2), we consider the following improved/adjusted discrepancy 
for regularization purpose:

$$
D_{\lambda}(A, \mathcal{Q})=\min _{R \in \mathcal{Q}} \Delta_{\lambda}(A, R), \quad \lambda>0,
$$

where $\mathcal{Q}$ is a set consisting of positive semi-definite matrices with a certain structure in certain candidate class, for example, the class considered in Lin et al. (2014). Consequently, we can deal with the regularization of correlation structures for high-dimension and low-sample-size (HDLSS) cases by solving the optimization problem (4) for each structure in the candidate class $\mathcal{Q}$.

It is likely that different values of $\lambda$ may lead to different regularized covariance estimators. We therefore need to select an optimal value of $\lambda$ via certain selection criteria. In the spirit of Cui et al. (2016), we propose to minimize the Frobenius-norm discrepancy when selecting the optimal $\lambda$. Hence our regularized covariance estimator takes the advantages of both entropy loss and Frobenius-norm discrepancy in this sense.

It is worth to mention that the entropy loss entropy functions $L(A, R)$ and $\Delta_{\lambda}(A, R)$ between matrices $A$ and $R$ are two special Bregman divergences with different generators (Dhillon and Tropp, 2007). Recently, using Bregman divergence to deal with statistics problems, such as estimation of models (Bauwens et al., 2016; Chrétien and Ortega, 2014) and matrix nearness problems (Dhillon and Tropp, 2007), has become increasingly common. The main advantage of using the special Bregman divergence $\Delta_{\lambda}(A, R)$ with an appropriate parameter $\lambda$ is that it works well in regularization for HDLSS data. In what follows, the notation $\Delta(A, R)$ instead of $\Delta_{\lambda}(A, R)$ is adopted for brevity while the parameter $\lambda$ is not emphasized.

This paper is organized as follows. In Section 2, we discuss the properties of the loss function adopted as a special Bregman divergence. In Section 3 we derive the globally optimal solution of the optimization problem (4) with a certain $\lambda$ for the first three correlation structures and provide an iterative algorithm for banded Toeplitz structure. In Section 4, we explain in details how to select the optimal value of $\lambda$, with which the optimal regularized estimator with a certain structure is obtained. In Section 5, some numerical simulations and real data analysis are provided for assessing the performance of the proposed regularization approach, especially for HDLSS situations. Section 6 gives some discussions and conclusions.

\section{Properties of the loss function (3)}

The loss function (3) between matrix $A$ and matrix $R$ agrees with the matrix Bregman divergence between $A$ and $R$ generated by a strictly convex function $\Phi(X)=-\log |X+\lambda I|$ with respect to positive semi-definite matrix $X \in \mathbb{R}^{p \times p}$ (Dhillon and Tropp, 2007). The KL divergence in (1) is a 
special case of the loss function (3) with $\lambda=0$ and the matrices $A$ and $R$ prescribed as positive definite matrices.

Assume that two positive semi-definite matrices $A$ and $R$ respectively have eigenpairs $\left(\alpha_{m}, \vec{\alpha}_{m}\right)$ and $\left(\beta_{m}, \vec{\beta}_{m}\right), m=1, \ldots, p$. Then the loss function (3) can be expressed as the weighted sum of the scalar divergences (Dhillon and Tropp, 2007)

$$
\Delta_{\lambda}(A, R)=\sum_{m=1}^{p} \sum_{n=1}^{p}\left|\left\langle\vec{\alpha}_{m}, \vec{\beta}_{n}\right\rangle\right|^{2} \delta_{\lambda}\left(\alpha_{m}, \beta_{n}\right),
$$

where

$$
\delta_{\lambda}(a, b)=(a+\lambda)^{-1}(b+\lambda)-\log \left((a+\lambda)^{-1}(b+\lambda)\right)-1, \quad \lambda>0
$$

is the scalar Bregman divergence from $a \geq 0$ to $b \geq 0$ generated by strictly convex function $\phi(x)=-\log (x+\lambda), x \geq 0$. This fact shows that the loss function (3) captures the difference of two matrices in their eigenvalues and eigenvectors.

\section{Regularization of correlation structures}

In this section, we discuss the regularization of correlation structures by solving the optimization problem (4), provided that the parameter $\lambda$ is given.

\subsection{Structure: $M A(1)$}

Consider a correlation coefficient matrix with the following structure

$$
R_{1}(c)=\left(\begin{array}{ccccc}
1 & c & 0 & \cdots & 0 \\
c & 1 & \ddots & \ddots & \vdots \\
0 & \ddots & \ddots & \ddots & 0 \\
\vdots & \ddots & \ddots & 1 & c \\
0 & \cdots & 0 & c & 1
\end{array}\right)
$$

where $-1 /(2 \cos (\pi /(p+1))) \leq c \leq 1 /(2 \cos (\pi /(p+1)))$ that ensures the matrix $R_{1}(c)$ is positive semi-definite. It can be rewritten as

$$
R_{1}(c)=I+c T_{1}
$$


where $T_{1}$ is a symmetric matrix with the elements of the first superdiagonal and subdiagonal being 1 and all others 0 .

The eigenvalues of $R_{1}(c)$ are

$$
\mu_{i}=1+2 c s_{i}, i=1, \ldots, p
$$

where $s_{i}=\cos (i \pi /(p+1))$. Then, the loss function $\Delta\left(A, R_{1}(c)\right)$ with respect to $c$ can be rewritten as

$$
f(c)=c \operatorname{tr}\left((A+\lambda I)^{-1} T_{1}\right)-\sum_{i=1}^{p} \log \left(\lambda+1+2 c s_{i}\right)+\operatorname{tr}(A+\lambda I)^{-1}+\log \operatorname{det}(A+\lambda I)-p,
$$

and the optimization problem (4) can be rewritten as

$$
\min _{-\frac{1}{2 s_{1}} \leq c \leq \frac{1}{2 s_{1}}} f(c) .
$$

Denote $d f(c) / d c$ as $g(c)$. It follows that

$$
g(c)=\operatorname{tr}\left((A+\lambda I)^{-1} T_{1}\right)-\sum_{i=1}^{p} \frac{2 s_{i}}{\lambda+1+2 c s_{i}} .
$$

Theorem 3.1: The optimization problem (7) is strictly convex. Moreover,

(a) If $g\left(-1 /\left(2 s_{1}\right)\right) g\left(1 /\left(2 s_{1}\right)\right)<0$, then the globally optimal solution $\hat{c}$ is the unique solution of equation $g(c)=0$.

(b) If $g\left(-1 /\left(2 s_{1}\right)\right) g\left(1 /\left(2 s_{1}\right)\right) \geq 0$, then the globally optimal solution $\hat{c}$ satisfies $f(\hat{c})=$ $\min \left\{f\left(-1 /\left(2 s_{1}\right)\right), f\left(1 /\left(2 s_{1}\right)\right)\right\}$.

Proof. It is easy to see

$$
\frac{d^{2} f(c)}{d c^{2}}=\frac{d g(c)}{d c}=\sum_{i=1}^{p}\left(\frac{2 s_{i}}{\lambda+1+2 c s_{i}}\right)^{2}>0 .
$$

Therefore, for $c \in\left[-1 /\left(2 s_{1}\right), 1 /\left(2 s_{1}\right)\right], f(c)$ is strictly convex and $g(c)$ is monotonic.

If $g\left(-1 /\left(2 s_{1}\right)\right) g\left(1 /\left(2 s_{1}\right)\right)<0$, then there exists only one solution $\hat{c} \in\left(-1 /\left(2 s_{1}\right), 1 /\left(2 s_{1}\right)\right)$ such that $g(c)=0$, and then $\hat{c}$ is the globally optimal solution of problem (7). Otherwise, the object function $f(c)$ is monotonic and then the globally optimal solution to (7) is corresponding to the smaller one of $f\left(-1 /\left(2 s_{1}\right)\right)$ and $f\left(1 /\left(2 s_{1}\right)\right)$.

Theorem 3.1 shows how to exactly find the globally optimal solution of optimization problem (7). 


\subsection{Structure: Compound symmetry}

Consider a correlation coefficient matrix with the following structure

$$
R_{2}(c)=\left(\begin{array}{ccccc}
1 & c & c & \cdots & c \\
c & 1 & \ddots & \ddots & \vdots \\
c & \ddots & \ddots & \ddots & c \\
\vdots & \ddots & \ddots & 1 & c \\
c & \cdots & c & c & 1
\end{array}\right)
$$

where $-1 /(p-1) \leq c \leq 1$ that ensures the matrix $R_{2}(c)$ is positive semi-definite. It can be rewritten as

$$
R_{2}(c)=I+c\left(e e^{T}-I\right)
$$

where $e=(1, \ldots, 1)^{T} \in \mathbb{R}^{m}$.

The eigenvalues of $R_{2}(c)$ are given by

$$
\begin{aligned}
& \mu_{1}=1+(p-1) c \\
& \mu_{i}=1-c, i=2, \ldots, p .
\end{aligned}
$$

Therefore, the loss function $\Delta\left(A, R_{2}(c)\right)$ with respect to $c$ can be rewritten as

$$
\begin{aligned}
f(c)= & c \operatorname{tr}\left((A+\lambda I)^{-1}\left(e e^{T}-I\right)\right)-\log (\lambda+1+(p-1) c)-(p-1) \log (\lambda+1-c) \\
& +\operatorname{tr}\left((A+\lambda I)^{-1}\right)+\log \operatorname{det}(A+\lambda I)-p,
\end{aligned}
$$

and the optimization problem (4) can be rewritten as

$$
\min _{-\frac{1}{p-1} \leq c \leq 1} f(c)
$$

Denote $d f(c) / d c$ as $g(c)$. Then it follows that

$$
g(c)=\operatorname{tr}\left((A+\lambda I)^{-1}\left(e e^{T}-I\right)\right)-\frac{p-1}{\lambda+1+(p-1) c}+\frac{p-1}{\lambda+1-c} .
$$

Theorem 3.2: The optimization problem (9) is strictly convex. Moreover,

(a) If $g(-1 /(p-1)) g(1)<0$, then the globally optimal solution $\hat{c}$ is the unique solution of equation $g(c)=0$ in $(-1 /(p-1), 1)$. 
(b) If $g(-1 /(p-1)) g(1) \geq 0$, then the globally optimal solution $\hat{c}$ satisfies $f(\hat{c})=\min \{f(-1 /(p-$ $1)), f(1)\}$.

Theorem 8 can be proven similarly to Theorem 3.1. It shows that the globally optimal solution to optimization problem (9) can be accurately attained.

\subsection{Structure: $A R(1)$}

Consider a correlation matrix with following structure

$$
R_{3}(c)=\left(\begin{array}{ccccc}
1 & c & c^{2} & \cdots & c^{p-1} \\
c & 1 & c & \cdots & c^{p-2} \\
\vdots & \ddots & \ddots & \ddots & \vdots \\
c^{p-2} & \ddots & \ddots & 1 & c \\
c^{p-1} & c^{p-2} & \cdots & c & 1
\end{array}\right)
$$

where $-1 \leq c \leq 1$ that ensures the matrix $R_{3}(c)$ is positive semi-definite. It can be rewritten as

$$
R_{3}(c)=\sum_{i=0}^{p-1} c^{i} T_{i},
$$

where $T_{0}=I$ and $T_{i}(i=1, \ldots, p-1)$ is a symmetric matrix with ones on the $i$-th superdiagonal and subdiagonal and zeros elsewhere.

Denote $\Delta\left(A, R_{3}(c)\right)$ as $f(c)$. The optimization problem (4) can be rewritten as

$$
\min _{c \in[-1,1]} f(c)
$$

where $f(c)$ is continuous and bounded below over a bounded set. By observing the plot of function $f(c)$ over $[-1,1]$, it is concluded that this is a non-convex problem.

We therefore propose to use a direct search method to solve problem (11), which is effective for one-dimension optimization problems with bounded regions. The algorithm is presented as follows:

Algorithm 3.1 (Direct search method): Given $m$,

(a) Calculate $f\left(c_{i j}\right)$, where $c_{i j}=(-1)^{j} i / m, i=0, \ldots, m, j=0,1$.

(b) Find the minimum in the set of $\left\{f\left(c_{i j}\right): i=0, \ldots, m, j=0,1\right\}$, which corresponds to the globally optimal solution to problem (11). 


\subsection{Structure: Banded Toeplitz}

Consider a correlation coefficient matrix with following structure

$$
R_{4}(\mathbf{c})=\left(\begin{array}{ccccc}
1 & c_{1} & c_{2} & \cdots & c_{p} \\
c_{1} & 1 & c_{1} & \cdots & c_{p-1} \\
c_{2} & c_{1} & 1 & \cdots & c_{p-2} \\
\vdots & \ddots & \ddots & \ddots & \vdots \\
c_{p} & c_{p-1} & \cdots & c_{1} & 1
\end{array}\right)
$$

where $\mathbf{c}=\left(c_{1}, c_{2}, \ldots, c_{p-1}\right)^{T}$ belongs to the following set (Parter, 1962)

$$
\Omega=\left\{\mathbf{c}=\left(c_{1}, c_{2}, \ldots, c_{p-1}\right)^{T} \in \mathbb{R}^{p-1}: 1+2 \sum_{k=1}^{p-1} c_{k} \cos (k t) \geq 0 \text { and } \not \equiv 0 \text { for all } t \in \mathbb{R}\right\},
$$

which ensures the matrix $R_{4}(\mathbf{c})$ is definite positive. It can be rewritten as

$$
R_{4}(\mathbf{c})=I+\sum_{i=1}^{p-1} c_{i} T_{i}
$$

Denote $f(\mathbf{c})=\Delta\left(A, R_{4}(\mathbf{c})\right)$. Then the optimization problem in (4) can be rewritten as

$$
\min _{\mathbf{c} \in \Omega} f(\mathbf{c}) .
$$

Theorem 3.3: The optimization problem (14) is convex.

Proof. Firstly, it is well known that $\Delta(A, R)$ is strictly convex function of $R$ (Dhillon and Tropp, $2007)$ and $R_{4}(\mathbf{c})=I+\sum_{i=1}^{p-1} c_{i} T_{i}$ is an affine map of $\mathbf{c}$. Then the function $f(\mathbf{c})$ is strictly convex in c because the composition of convex function with an affine mapping preserves convexity. Secondly, it is easy to prove that $\Omega$ is a convex set. This theorem thus follows.

In general, Newton's method works well for convex optimization problems. Here, Newton's method with backtracking line search (Boyd and Vandenberghe, 2004) is applied to solving problem (14). The gradient $g$ and Hessian matrix $H$ of $f$ are given as follows:

$$
\begin{gathered}
g(i)=\nabla_{c_{i}} f=\operatorname{tr}\left(T_{i}\left((A+\lambda I)^{-1}-\left(R_{4}(\mathbf{c})+\lambda I\right)^{-1}\right)\right), i=1, \ldots, p-1, \\
H(i, j)=\nabla_{c_{i} c_{j}} f=\operatorname{tr}\left(T_{i}\left(R_{4}(\mathbf{c})+\lambda I\right)^{-1} T_{j}\left(R_{4}(\mathbf{c})+\lambda I\right)^{-1}\right), i, j=1, \ldots, p-1 .
\end{gathered}
$$

We provide the following search algorithm for solving problem (14). 
Algorithm 3.2 (Newton's method with backtracking line search): Given an initial point $\mathbf{c} \in \Omega$ given by (13) and a tolerance $\epsilon$, repeat:

(a) Calculate the gradient $g$ given by (15) and the Hessian matrix $H$ given by (16) at the point $\mathbf{c}$.

(b) Compute the Newton step $\Delta \mathbf{c}$ and decrement $r^{2}$ :

$$
\begin{aligned}
\Delta \mathbf{c} & :=-H^{-1} g, \\
r^{2} & :=g^{T} H g .
\end{aligned}
$$

(c) Stopping criterion:

$$
\text { quit if } r^{2} / 2 \leq \epsilon
$$

(d) Backtracking line search: given two parameters $\alpha \in(0,0.5)$ and $\beta \in(0,1)$, do

$$
\begin{aligned}
& t:=1 \\
& \text { while } \mathbf{c}+t \Delta \mathbf{c} \notin \Omega, \\
& \qquad t:=\beta t \\
& \text { while } f(\mathbf{c}+t \Delta \mathbf{c})>f(\mathbf{c})+\alpha t g^{T} \Delta \mathbf{c}, \\
& \qquad t:=\beta t .
\end{aligned}
$$

(e) Update c:

$$
\mathbf{c}:=\mathbf{c}+t \Delta \mathbf{c}
$$

The globally optimal solution to optimization problem (14) can be attained using Algorithm 3.2, of which the number of Newton iterations required is described in detail in Lin et al. (2014). In the following simulations, the absolute convergence tolerance of the problem is always set as $\epsilon=10^{-10}$.

\section{The selection of parameter $\lambda$}

As proposed before, the loss function (3) is used to deal with the regularization of correlation structures for HDLSS cases. While it is not really efficient for some small $\lambda$ as it involves computing the inverse of $A+\lambda I$ (if a matrix is ill-conditioned or nearly singular, its inverse would be inaccurately computed (Stewart, 1998)). So some simulation experiments were made to investigate how the parameter $\lambda$ affects the estimation accuracy of structural correlation matrix. In what follows, 
the notation $\hat{R}_{i}^{\lambda}$ means the regularized estimator with structure $R_{i}$ achieved when the parameter $\lambda$ is given.

A typical simulation result showing the effect of $\lambda$ is provided in Table 1, in which all results are averaged over 1000 replications. The simulated data are generated from the multivariate normal distribution $N(0, R)$, where $R$ is exactly the correlation matrix with the Toeplitz structure $B_{4}$ and is randomly generalized. The criterion value $L\left(R, \hat{R}_{4}^{\lambda}\right)$ is the KL divergence mentioned in (1), evaluating the estimation accuracy of $\hat{R}_{4}^{\lambda}$, between the real correlation matrix $R$ and the regularized estimator $\hat{R}_{4}^{\lambda}$.

Table 1. The estimated entropy loss (standard errors in parentheses) for different parameter $\lambda$

\begin{tabular}{ccccccccc}
\hline$\lambda$ & 0.01 & 0.1 & 0.5 & 1 & 5 & 10 & 100 \\
\hline$L\left(R, \hat{R}_{4}^{\lambda}\right)$ & $118.39(12.96)$ & $16.54(3.16)$ & $1.75(0.25)$ & $1.25(0.16)$ & $1.13(0.16)$ & $1.08(0.16)$ & $1.14(0.19)$ & $1.16(0.20)$ \\
\hline
\end{tabular}

Remark 4.1: A real $p \times p$ Toeplitz matrix can be embedded in a $(2 p-1) \times(2 p-1)$ circulant matrix, whose eigenvector matrix is the normalized Fourier transform matrix and whose eigenvalues are $z_{0}, z_{1}, \ldots, z_{p-2}, z_{p-1}, z_{p-1}, z_{p-2}, \ldots, z_{1}$. In this paper, for generating a positive definite circulant matrix $C, z_{0}$ is set as 1 , and $z_{1}, \ldots, z_{p-1}$ are randomly generated from the uniform distribution on the open interval $(0,1)$. Then we obtain a positive Toeplitz matrix $R^{\prime}=[I, 0] C[I, 0]^{T}$. Finally, a correlation matrix $R=\operatorname{diag}\left(R^{\prime}\right)^{\frac{1}{2}} R^{\prime} \operatorname{diag}\left(R^{\prime}\right)^{\frac{1}{2}}$ is generated.

The results in Table 1 suggest that any $\lambda$ not being too small could yield a good regularized estimate. While, in applying this method, one will usually need to select a $\lambda$ from a set of not too small $\lambda$. A natural choice of the criterion of electing $\lambda$ maybe minimizing the KL divergence from the true correlation matrix $R$ to the regularized correlation matrix $\hat{R}_{i}^{\lambda}$. As $R$ is unknown, it is usually replaced by the sample correlation matrix $A$. In HDLSS cases, the inverse of $A$ in the KL divergence criterion does not always exist because $A$ may be singular, thus other appropriate criterion for electing $\lambda$ is needed.

It is not uncommon to use the Frobenius-norm between the true covariance and the estimated covariance to evaluate the estimation accuracy because of its simplicity. Here, as the true correlation matrix is unknown, the Frobenius-norm between the given correlation matrix and the regularized correlation matrix

$$
F\left(A, \hat{R}_{i}^{\lambda}\right)=\operatorname{tr}\left(A-\hat{R}_{i}^{\lambda}\right)\left(A-\hat{R}_{i}^{\lambda}\right)^{T}, \quad i=1,2,3,4
$$


is used as the evaluation criterion (Cui et al., 2016). In other words, we take

$$
\lambda_{0}=\arg \min _{\lambda} F\left(A, \hat{R}_{i}^{\lambda}\right), \quad i=1,2,3,4
$$

as the optimal $\lambda$ for regularizing the given correlation matrix $A$ when using structure $R_{i}$. The corresponding optimal regularized estimator with structure $R_{i}$ is denoted as $R_{i}^{\lambda_{0}}$ or $\hat{R}_{i}$ for brevity.

In the following, some simulation results are provided in Table 2 to show the reasonableness of evaluation criterion (17) of $\lambda$, in which all results of each experiment are averaged over 1000 replications. This table contains results of 5 simulation experiments. In each experiment, the true correlation matrix $R$ with the general Toeplitz structure $R_{4}$ is newly randomly generated as this in Remark 4.1, and the simulated data is generated from multivariate normal distribution $N(0, R)$. In Table $2, v$ is defined by

$$
v=\left|L\left(R, \hat{R}_{4}^{\lambda_{0}}\right)-L\left(R, \hat{R}_{4}^{\lambda_{1}}\right)\right|,
$$

where

$$
\lambda_{1}=\arg \min _{\lambda} L\left(R, \hat{R}_{4}^{\lambda}\right)
$$

which means $\lambda_{1}$ is identified by minimizing the KL divergence between the true correlation matrix $R$ and the regularized correlation matrix $\hat{R}_{4}^{\lambda}$.

Table 2. Differences between two regularized estimators by using the criteria (17) and (19) in terms of closeness to the true correlation matrix $R$ (standard errors in parentheses)

\begin{tabular}{cccccc}
\hline No. & 1 & 2 & 3 & 4 & 5 \\
\hline$v$ & $0.06(0.05)$ & $0.09(0.07)$ & $0.07(0.06)$ & $0.07(0.05)$ & $0.07(0.05)$ \\
\hline
\end{tabular}

The measurement $v$ in Table 2 shows the differences between estimators $\hat{R}_{4}^{\lambda_{0}}$ and $\hat{R}_{4}^{\lambda_{1}}$. Observing that all the values in this table appear very small (about $5 \%$ of $L\left(R, \hat{R}_{4}^{\lambda_{1}}\right)$ ), we conclude that the two estimators are nearly the same in the sense of their closeness to the real correlation matrix $R$. Thus, we believe it is reasonable to replace the criterion (19) with the criterion (17) for choosing an appropriate $\lambda$.

Remark 4.2: It is noted that the loss function (3) tends to zero as $\lambda$ tends to positive infinity. We therefore would not suggest to use the criterion of minimizing (3) to select the optimal $\lambda$, as it is not reasonable and reliable to directly minimize loss function (3) with respect to $\lambda$. 


\section{Numerical experiments}

In this section, we conduct simulation studies and real data analysis in order to show the performance of the proposed method that regularizes the sample correlation matrices for HDLSS data.

The numerical simulations are divided into two parts. One is for Gaussian data sets, and the other for scaled Gaussian data sets. All the methods are executed in MATLAB, where the rootfinding problem in Section 3.1 is solved by the function "fzero". In each experiment, the sample size is chosen as $n=100$, and the optimal value of $\lambda$ is selected in the interval from 1 to 200 with step 5 .

The notation and abbreviation for the results to be reported are summarized as follows:

$A$ : The sample correlation matrix;

$R$ : The real correlation matrix whose structure parameter involved is denoted as $c_{R}$;

$\hat{R}_{i}$ : The regularized estimator with structure $R_{i}$;

$L_{\hat{R}_{i} R}$ : The KL divergence between the true correlation matrix $R$ and the regularized correlation matrix $\hat{R}_{i}$ :

$$
L_{\hat{R}_{i} R}=\operatorname{tr}\left(R^{-1} \hat{R}_{i}\right)-\log \left(\operatorname{det}\left(R^{-1} \hat{R}_{i}\right)\right)-p
$$

$F_{R \hat{R}_{i}}:$ The root-mean-square error (RMSE) of $\hat{R}_{i}$ :

$$
F_{R \hat{R}_{i}}=\left(\operatorname{tr}\left(R-\hat{R}_{i}\right)\left(R-\hat{R}_{i}\right)^{T}\right)^{\frac{1}{2}}
$$

$F_{A \hat{R}_{i}}$ : The Frobenius-norm discrepancy between the matrices $A$ and $\hat{R}_{i}$ :

$$
F_{A \hat{R}_{i}}=\left(\operatorname{tr}\left(A-\hat{R}_{i}\right)\left(A-\hat{R}_{i}\right)^{T}\right)^{\frac{1}{2}}
$$

$L_{\text {diff }}$ : The KL divergence between the two best regularized estimators in terms of minimizing $F_{A \hat{R}_{i}}$ :

$$
L_{\text {diff }}=\operatorname{tr}\left(\hat{R}_{j}^{-1} \hat{R}_{k}\right)-\log \left(\operatorname{det}\left(\hat{R}_{j}^{-1} \hat{R}_{k}\right)\right)-p, \quad k<j .
$$

Remark 5.1: In all our simulations, the regularized estimators $\hat{R}_{i}$ are always positive definite. Thus, here $L_{\hat{R}_{i} R}$ and $L_{\text {diff }}$ can be normally calculated. 


\subsection{Experiments for Gaussian data sets}

We calculate sample correlation matrix $A$ by an $n \times p$ data matrix with columns randomly drawn from the multivariate normal distribution $N(0, \Sigma)$, where the covariance matrix $\Sigma$ is equal to its correlation matrix $R$. The correlation matrix $R$ has structure $R_{1}, R_{2}, R_{3}$ or $R_{4}$ stated above. For different structures of $R, n$ and $p$, different sample covariance matrices $A$ are formed. We choose different values of parameter $c$ for $R$ having the structure $R_{1}, R_{2}$ or $R_{3}$, while the correlation matrix $R$ with the structure $R_{4}$ is randomly generalized as Remark 4.1. To assess the performance of the proposed regularization method for HDLSS data, we consider $p=100,200,500$ respectively. For each combination of the above parameters, we run the simulation study for 100 times and report the averaged values of the results in Table 3 .

\subsection{Experiments for scaled Gaussian data sets}

In the above experiments, data are from Gaussian distribution, which is a general prerequisite in some researches. To stress that our method does not require a distribution assumption, similar experiments but for data from a scaled Gaussian distribution are carried out. In every experiments, the sample correlation matrix $A$ is calculated by iid observations $x_{1}, \ldots, x_{n}$, and $x_{i} \sim \sqrt{\tau} \nu$, where $\tau \sim \chi_{5}^{2}, \nu \sim N(0, \Sigma)$, and $\tau$ and $\nu$ are independent. In those experiments, the matrix $\Sigma$ is set to be equal to the correlation matrix $R$. The simulation setup of the true correlation matrix is the same as that of the Gaussian distribution considered above. We run each of the experiments for 100 times and report the averaged values in Table 4.

From Tables 3 and 4, we have the following observations:

i) The regularized estimators, having the same structure as the true correlation matrix $R$ and the Toeplitz structure $R_{4}$, is close to $R$ in terms of the KL divergence $L_{\hat{R}_{i} R}$ and Frobenius-norm $F_{\hat{R}_{i} R}$. It is not surprising for $\hat{R}_{4}$ to keep performing well because $R_{1}, R_{2}$ or $R_{3}$ are special Toeplitz structures.

ii) Note that the regularized estimators, having the same structure as the true correlation matrix $R$ and Toeplitz structure $R_{4}$, win out the other two regularized estimators in terms of minimizing the discrepancy $F_{A \hat{R}_{i}}$. Thus the observation made from the discrepancy $F_{A \hat{R}_{i}}$ is extremely important and useful in identifying the underlying structure.

iii) The two best regularized estimators, estimators having the same structure as the true correlation matrix $R$ and Toeplitz structure $R_{4}$, are close to each other in terms of KL divergence as $L_{\text {diff }}$ is always comparatively small.

iv) Our regularization approach works very well in regularizing sample correlation matrix even if 
Table 3. Simulation results for Gaussian data sets described in Section 5.1

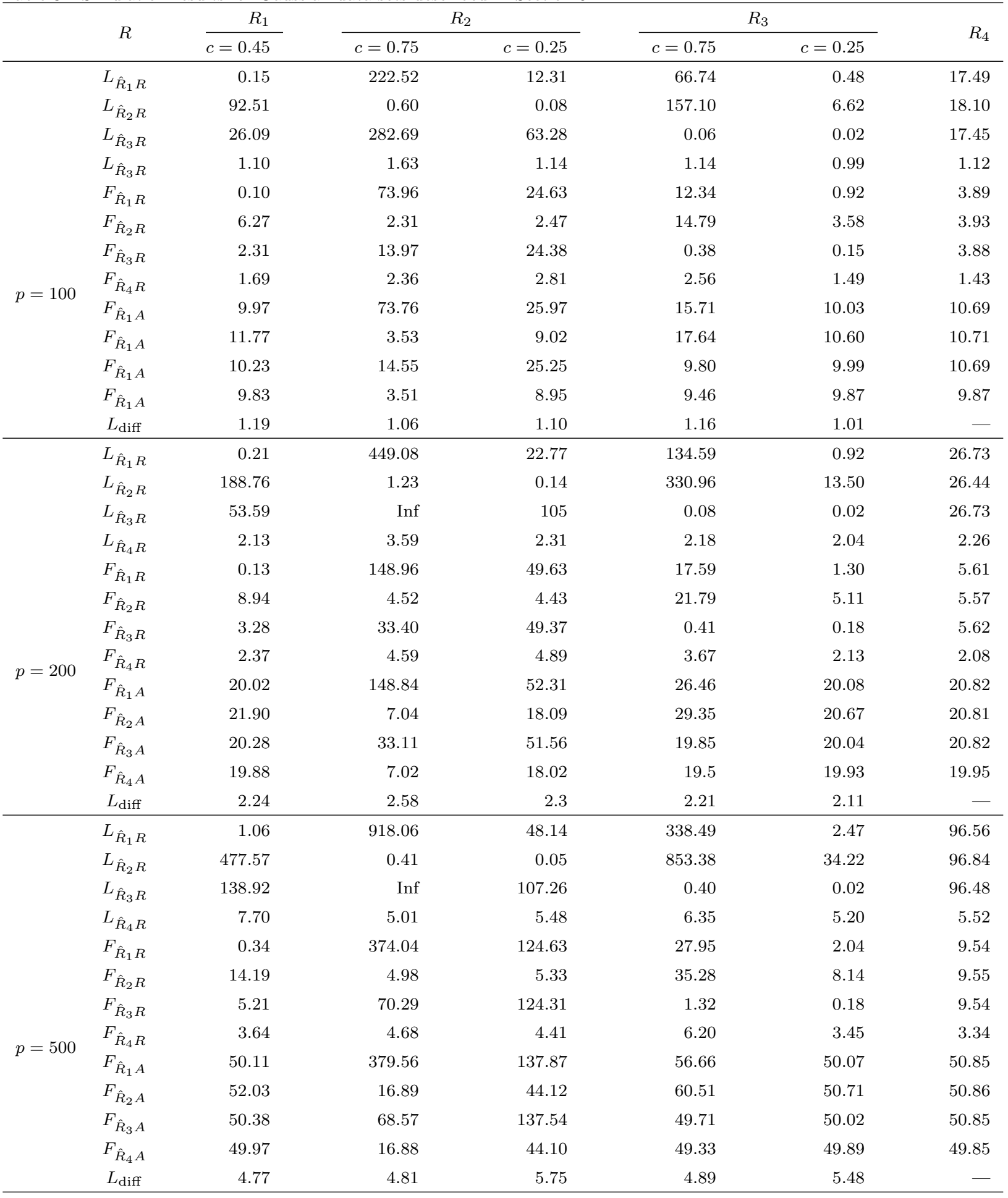

the dimension $p$ is far larger than the sample size $n$. The sample correlation matrix, though being a rather poor estimate of the population correlation matrix for high-dimension data, is still helpful in identifying the correct structure of the population correlation matrix. 
Table 4. Simulation results for non-Gaussian data sets described in Section 5.1

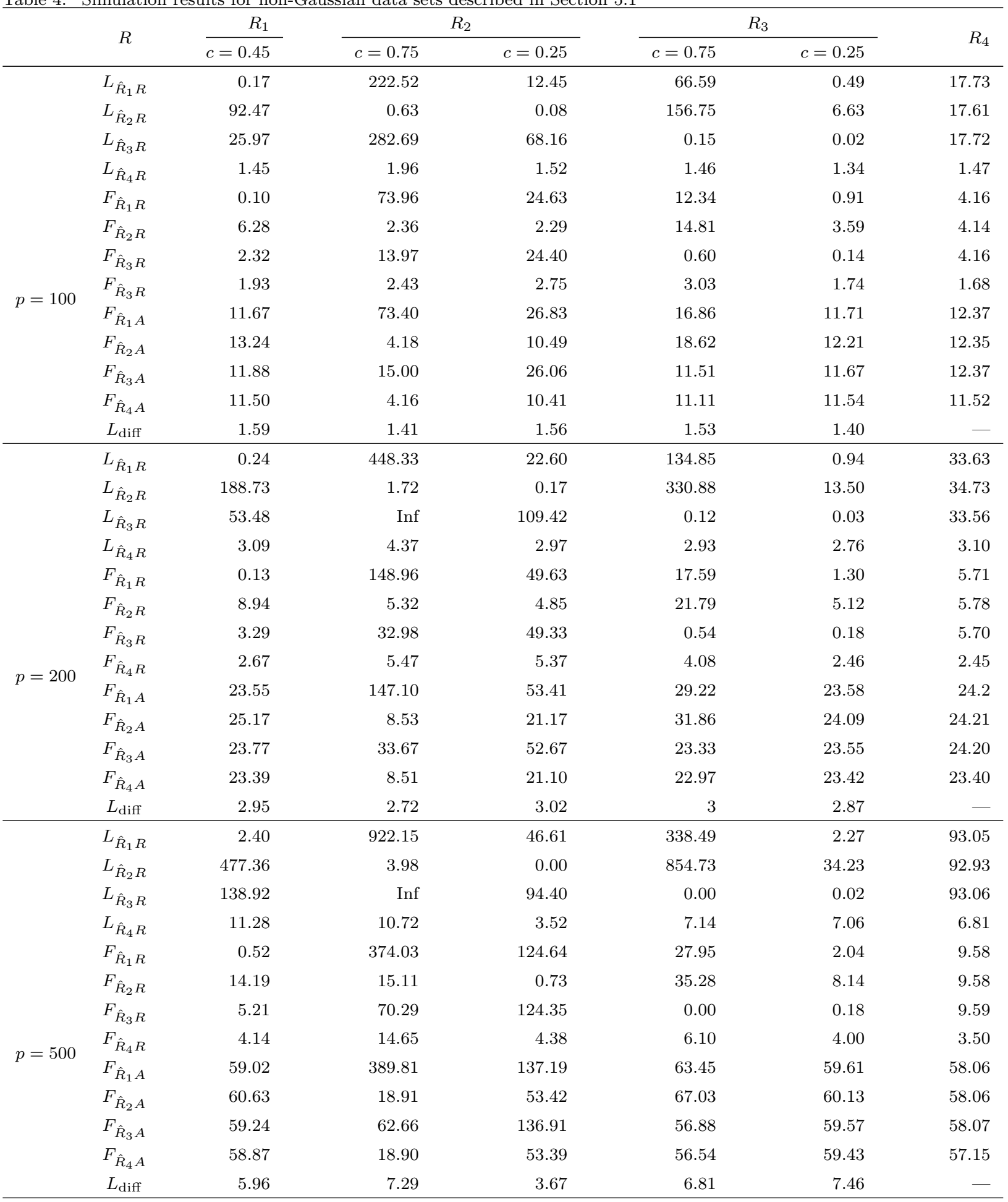

\subsection{Comparison with the regularization via the Frobenius-norm loss function}

In this subsection, we compare our regularized method with the common method in which the parameter vector $\mathbf{c}$ of a correlation matrix $R$ with the Toeplitz structure $R_{4}$ is estimated as follows:

$$
\hat{c}_{i}=\frac{1}{p-(i-1)} \sum_{s-t=i \frac{1}{1} 5} A_{s t}, i=1, \ldots, p-1,
$$


where $A_{s t}$ is the $(s, t)$-th element of the sample correlation matrix $A$. In fact, $(20)$ is derived by solving the optimization problem

$$
\hat{\mathbf{c}}=\arg \min _{\mathbf{c}}\|R-A\|_{F}^{2}, \text { s.t. } R=I+\sum_{i=1}^{p-1} c_{i} T_{i} .
$$

We generated an $n \times p$ data matrix with columns randomly drawn from the multi-variate normal distribution $N(0, R)$, where $R$ is precisely the correlation matrix and with the Toeplitz structure $R_{4}$. Here, $R$ is randomly generated as Remark 4.1 , and $n=10$.

Results in Table 5 conclude the RMSE and operator norm loss of the two regularized estimators with structure $R_{4}$ by the two methods. In the table, the entropy method is our regularized method, and the Frobenius-norm method is the regularized method as indicated in (20).

Table 5. Comparison of average matrix losses over 1000 replications (standard errors in parentheses)

\begin{tabular}{cccccc}
\hline & \multicolumn{2}{c}{$p=50$} & & \multicolumn{2}{c}{$p=100$} \\
\cline { 2 - 3 } & RMSE & Operator norm loss & & RMSE & Operator norm loss \\
\hline Entropy method & $2.47(0.24)$ & $0.79(0.38)$ & & $3.22(0.19)$ & $0.58(0.12)$ \\
\hline Frobenius-norm method & $3.37(0.40)$ & $1.79(1.10)$ & & $4.89(0.41)$ & $2.13(0.98)$ \\
\hline
\end{tabular}

From the results in Table 5, we can see that our regularized method outperforms the regularized method (20) in terms of the RMSE and operator norm loss.

\subsection{Real data analysis}

This subsection provides some analyses about a real data set that was given and analyzed in Verbyla and Cullis (1990). The data is from an experiment conducted to examine the relationship between legumes and beef productions. Four different treatments on legumes were designed, and for each treatment, legumes in four identical paddocks was cultivated using it. There were 16 paddocks in total. In each paddock, there are two Hereford weaner heifers. The study lasted for 4 years, with 17 measurements of live-weight (mean of 2 animals) per paddock taken at equal time intervals for each of the 2-year periods. The heifers were replaced after the first 2-year period.

As analysed in Verbyla and Cullis (1990), for each 2-year period, the multivariate regression mode is used. In other words, for each 2-year period, we have $Y \sim N\left(X T, I_{n} \otimes \sigma^{2} R\right)$ with $n=16$ 
and $p=16$, where

$$
X=\left(\begin{array}{llll}
1_{4} & 1_{4} & 0_{4} & 0_{4} \\
1_{4} & 0_{4} & 1_{4} & 0_{4} \\
1_{4} & 0_{4} & 0_{4} & 0_{4} \\
1_{4} & 0_{4} & 0_{4} & 1_{4}
\end{array}\right)
$$

with the $1_{4}$ and $0_{4}$ being 4 -dimensional vectors of ones and zeros respectively, and $\sigma^{2}$ is the error variance. Also it was said in Verbyla and Cullis (1990) that considering the long-term nature of the experiment, using an unstructured or fully parameterized covariance matrix is likely to be inefficient and modelling the covariance structure is desirable for parsimony and efficiency. Thus our regularized method is applied here to regularize the structure of the correlation matrix $R$.

The sample correlation matrix is $A=\operatorname{diag}(S)^{\frac{1}{2}} S \operatorname{diag}(S)^{\frac{1}{2}}$ where $S=(Y-X \hat{T})^{T}(Y-X \hat{T}) / n$ and $\hat{T}=\left(X^{T} X\right)^{-1} X^{T} Y$ is the least-squares estimate of the regression coefficient matrix $T$. The results about regularizing the sample correlation matrix $A$ by our regularized method are reported in Table 6 .

Table 6. Results of regularizing the sample correlation matrix $A$ by our regularized method.

\begin{tabular}{cccccc}
\hline & $F_{A \hat{R}_{1}}$ & $F_{A \hat{R}_{2}}$ & $F_{A \hat{R}_{3}}$ & $F_{A \hat{R}_{4}}$ & \\
\hline Years 1 and 2 & 5.04 & 5.16 & 5.16 & 4.92 & 0.90 \\
\hline Years 3 and 4 & 4.58 & 4.67 & 4.68 & 4.42 & 0.91 \\
\hline
\end{tabular}

The simulation results in Sections 5.1 and 5.2 show that the two regularized estimators, winning out the other two regularized estimators in terms of minimizing the discrepancy $F_{A \hat{R} i}$, are with the general Toeplitz structure $R_{4}$ and the same structure as the real correlation matrix. Here, we can see that, from Table 6 , for each 2 -year period, the regularized estimators $\hat{R}_{1}$ and $\hat{R}_{4}$ win out in terms of minimizing the discrepancy $F_{A \hat{R} i}$, and also $L_{\text {diff }}$ measuring the difference between the two best regularized estimators is not large. Thus we claim that both the correlation structures of the two 2-year periods tend to be $R_{1}(\mathrm{MA}(1))$. In addition, the parameters of the regularized estimators with structure $R_{1}$ in both the 2 -year periods are respectively -0.2059 and -0.1718 . This is consistent with the conclusion in Verbyla and Cullis (1990).

\section{Conclusions and Discussions}

In this paper, for HDLSS data, an improved discrepancy was proposed to regularize the structure of a correlation matrix whose underlying correlation structure comes from an available class including tri-diagonal Toeplitz, compound symmetry, AR(1) and banded Toeplitz. The discrepancy 
is measured by an entropy loss function in which the two matrix variates are added by a multiple of identity matrix so as to deal with the singular or near singular matrices, where the scale of this multiple is estimated using Frobenius-norm discrepancy. More generally, if the eigenvalues of matrices are too large or too small, then the matrix Bregman divergences generated by a strictly convex function $F(W)=\log \operatorname{det}\left(\lambda_{1} W+\lambda_{2} I\right)$ with two appropriate positive real numbers $\lambda_{1}$ and $\lambda_{2}$ can be adopted.

In this paper, we only consider the class of correlation structures that only contains four candidates. In statistics, there are many other common correlation structures, such as linearly structured correlation, factor analytic, and Hankel structures. Dealing with more structures is significant but challenging. We will report the development in our follow-up work.

\section{Acknowledgments}

This work was supported in part by the National Natural Science Foundation of China under Grant 61374027 and the Program for Changjiang Scholars and Innovative Research Team in University under Grant IRT_16R53.

\section{References}

Asl, B. M. and Mahloojifar, A. (2012). A low-complexity adaptive beamformer for ultrasound imaging using structured covariance matrix. IEEE Transactions on Ultrasonics, Ferroelectrics, and Frequency Control, 59(4):660-667.

Bauwens, L., Grigoryeva, L., and Ortega, J.-P. (2016). Estimation and empirical performance of non-scalar dynamic conditional correlation models. Computational Statistics and Data Analysis, 100:17-36.

Boyd, S. and Vandenberghe, L. (2004). Convex Optimization. Cambridge University Press, Cambridge, UK.

Chrétien, S. and Ortega, J.-P. (2014). Multivariate GARCH estimation via a Bregman-proximal trust-region method. Computational Statistics and Data Analysis, 76:210-236.

Cui, X., Li, C., Zhao, J., Zeng, L., Zhang, D., and Pan, J. (2016). Covariance structure regularization via Frobenius-norm discrepancy. Linear Algebra and its Applications, 510:124-145.

Daniels, M. J. and Kass, R. E. (2001). Shrinkage estimators for covariance matrices. Biometrics, $57(4): 1173-1184$. 
Dhillon, I. S. and Tropp, J. A. (2007). Matrix nearness problems with Bregman divergences. SIAM Journal on Matrix Analysis and Applications, 29(4):1120-1146.

Feng, S., Lian, H., and Xue, L. (2016). A new nested Cholesky decomposition and estimation for the covariance matrix of bivariate longitudinal data. Computational Statistics and Data Analysis, 102:98-109.

Jennrich, R. I. and Schluchter, M. D. (1986). Unbalanced repeated-measures models with structured covariance matrices. Biometrics, 42(4):805-820.

Kang, B., Monga, V., and Rangaswamy, M. (2015). Computationally efficient Toeplitz approximation of structured covariance under a rank constraint. IEEE Transactions on Aerospace and Electronic Systems, 51(1):775-785.

Kim, B. and Lee, S. (2013). Robust estimation for the covariance matrix of multivariate time series based on normal mixtures. Computational Statistics and Data Analysis, 57(1):125-140.

Lin, L., Higham, N. J., and Pan, J. (2014). Covariance structure regularization via entropy loss function. Computational Statistics and Data Analysis, 72:315-327.

Meyer, K. (2009). Factor-analytic models for genotype $\times$ environment type problems and structured covariance matrices. Genetics Selection Evolution, 41(1):1-11.

Parter, S. V. (1962). An observation on the numerical solution of difference equations and a theorem of Szegö. Numerische Mathematik, 4(1):293-295.

Pascal, F., Chitour, Y., Ovarlez, J.-P., Forster, P., and Larzabal, P. (2008). Covariance structure maximum-likelihood estimates in compound Gaussian noise: Existence and algorithm analysis. IEEE Transactions on Signal Processing, 56(1):34-48.

Shults, J. and Chaganty, N. R. (1998). Analysis of serially correlated data using quasi-least squares. Biometrics, 54(4):1622-1630.

Soloveychik, I., Trushin, D., and Wiesel, A. (2016). Group symmetric robust covariance estimation. IEEE Transactions on Signal Processing, 64(1):244-257.

Soloveychik, I. and Wiesel, A. (2014). Tyler's covariance matrix estimator in elliptical models with convex structure. IEEE Transactions on Signal Processing, 62(20):5251-5259.

Stewart, G. (1998). On the adjugate matrix. Linear Algebra and its Applications, 283(1):151-164. Sun, Y., Babu, P., and Palomar, D. P. (2015). Robust estimation of structured covariance matrix for heavy-tailed elliptical distributions. IEEE Transactions on Signal Processing, 64(14):3576-3590. van der Leeden, R., Vrijburg, K., and de Leeuw, J. (1996). A review of two different approaches for the analysis of growth data using longitudinal mixed linear models: comparing hierarchical linear regression (ML3, HLM) and repeated measures designs with structured covariance matrices (BMDP5V). Computational Statistics and Data Analysis, 21(5):583-605. 
Verbyla, A. P. and Cullis, B. R. (1990). Modelling in repeated measures experiments. Applied Statistics, 39(3):341-456.

Wang, Y.-G. and Carey, V. (2003). Working correlation structure misspecification, estimation and covariate design: implications for generalised estimating equations performance. Biometrika, 90(1):29-41.

Zhou, L., Takane, Y., and Hwang, H. (2016). Dynamic GSCANO (generalized structured canonical correlation analysis) with applications to the analysis of effective connectivity in functional neuroimaging data. Computational Statistics and Data Analysis, 101:93-109. 